\title{
Evaluation of Rapid stool antigen test for the diagnosis of Helicobacter pylori infection in patients with dyspepsia
}

\author{
Salma Khatun ${ }^{1}$, Fahmida Rahman ${ }^{1}$, Khandaker Shadia ${ }^{1}$, Indrajit Kumar Dutta ${ }^{2}$, Mohammad \\ Nazmul Hoq², Farjana Akter ${ }^{1}$, Jalaluddin Ashraful Haq ${ }^{1}$ \\ ${ }^{1}$ Department of Microbiology, Ibrahim Medical College, Dhaka; ${ }^{2}$ Department of Gastroenterology, \\ Hepatobiliary and Pancreatic Diseases, Bangladesh Institute of Research and Rehabilitation in Diabetes, \\ Endocrine and Metabolic Disorders, Dhaka
}

\begin{abstract}
Background and objectives: Several diagnostic assays are used for the detection of Helicobacter pylori infection in suspected peptic ulcer cases. H. pylori stool antigen test is a non-invasive method for the detection of active infection. The present study has evaluated the efficacy of rapid stool antigen test to diagnose $H$. pylori infection in patients with dyspepsia.

Materials and methods: Adult patients with complains of dyspepsia attending the Department of Gastroenterology, Hepatobiliary and Pancreatic Diseases (GHPD) of BIRDEM hospital for endoscopy were included. Gastric biopsy, blood and stool samples were obtained from each participant after informed written consent. Rapid urease test (RUT), serum H. pylori immunoglobulin A (IgA) and IgG and rapid $H$. pylori stool antigen (HpSAg) tests were performed. Only stool samples were obtained from 31 neonates aged 1 to 30 days as negative control for HpSAg test.

Results: A total of 91 adult patients with complain of dyspepsia were included in the study. Out of 91 cases, $17(18.7 \%)$ and $74(81.3 \%)$ had peptic ulcer and erosion respectively. HpSAg was positive in $63.7 \%$ cases compared to $42.9 \%$ and $62.6 \%$ respectively by RUT and IgA. The rate of HpSAg positivity was significantly higher $(\mathrm{p}<0.05)$ in ulcer compared to erosion cases. HpSAg test was positive in all (100\%) RUT positive cases. Combination of HpSAg test and IgA yielded highest positive result in both ulcer $(82.4 \%)$ and erosion (84\%) cases. H. pylori $\mathrm{IgG}$ was positive in all cases.
\end{abstract}

Conclusion: The study has demonstrated that HpSAg test is an effective and non-invasive diagnostic tool to detect active $H$. pylori infection in suspected dyspeptic patients.

IMC J Med Sci 2016; 10(2): 39-44

\section{Introduction}

Helicobacter pylori is known to be associated with peptic ulcer diseases. More than half of the world's population is infected with Helicobacter pylori, which is acquired almost always within the first 5 years of life [1]. Like other developing countries, the prevalence of $H$. pylori is very high in Bangladesh. The reported prevalence of $H$. pylori infection in adults is about $90 \%$ and more than $80 \%$ children become infected with $H$. pylori by the age of 6-9 years
$[2,3]$. Both invasive and non-invasive tests are available for the diagnosis of $H$. pylori infection. Invasive tests namely culture, staining, histology or rapid urease test (RUT) require biopsy specimens during endoscopy while noninvasive tests include serology, urea breath test (UBT) and stool antigen test ( $\mathrm{HpSAg})$.

Culture of the organism is the gold standard for diagnosis of $H$. pylori infection, but it is not available in most laboratories as it requires special growth condition and facilities [4]. Histology

\section{Address for Correspondence:}

Prof. Jalaluddin Ashraful Haq, Department of Microbiology, Ibrahim Medical College, 122 Kazi Nazrul Islam Avenue, Dhaka 1000, Bangladesh.Email: jahaq54@yahoo.com 
examination of biopsy material can provide important information about morphological features indicating severity of gastritis and evidence for dysplasia. However, the accuracy of histology may be variable due to density of $H$. pylori and sampling error and also subjective to experience of the pathologist [5]. Rapid urease test (RUT) is simple and provides quick results [6]. It is based on urease activity of $H$. pylori in biopsy sample taken during endoscopy. Sensitivity and specificity of RUT test depends on number of biopsies and bacterial load [7]. Any concomitant use of antibiotics reduces bacterial load, and may lead to false negative results in RUT, UBT and histology [8]. Furthermore, the presence of other microorganisms that produce urease can lead to false-positive results [9]. Serology is widely used for screening patients for $H$. pylori infection. It has a good sensitivity, is quick and relatively inexpensive, but has low specificity since antibody titers remain high for years after $H$. pylori eradication and have limited value to confirm $H$. pylori active infection [10]. The UBT provides a reliable noninvasive method for detection of $H$. pylori infection with sensitivity and specificity of $88-95 \%$ and $95 \%-100 \%$ respectively [7]. But UBT involves radio active materials and requires an expensive instrument, which is not always available in routine clinical laboratories.

As a gastrointestinal pathogen, H. pylori also appear in the stool. Stool tests have the advantage of being noninvasive and the specimen is easily obtainable. $H$. pylori stool antigen (HpSAg) assay has been proven to be clinically useful with sensitivities and specificities of more than $90 \%$ and is advantageous to confirm eradication [8]. It can be used as a routine diagnostic tool for $H$. pylori infection because it seems to overcome the limitations of the conventional invasive techniques. HpSAg test is suitable to use particularly in developing countries. Detection of $H$. pylori antigens in fecal sample might be useful for noninvasive diagnosis of $H$. pylori infection in children. HpSAg may be useful particularly in selection of the cases requiring endoscopic examination, in monitoring the response to treatment and in epidemiological studies [11]. Therefore, the aim of the present study was to evaluate the efficacy of a rapid immuno-chromatographic stool antigen test to diagnose $H$. pylori infection in dyspeptic patients.

\section{Materials and Methods}

Study population and sample collection: Ninety one adult patients with dyspeptic symptoms attending the Department of Gastrointestinal, Hepatobiliary and Pancreatic Diseases (GHPD) of BIRDEM General Hospital for diagnostic endoscopy were enrolled in the study. Patients treated with any antibiotics, colloidal bismuth compounds, proton pump inhibitors (PPI) or $\mathrm{H}_{2}$ blocker within the last four weeks were excluded from the study. Gastric biopsy specimen was obtained during endoscopy from every adult patient for detection of $H$. pylori infection by rapid urease test (RUT). In addition, stool (20-30 gm) and blood $(2.5 \mathrm{ml})$ samples were collected from each patient. Stool samples were tested for $H$. pylori antigen within 6 hours of collection. Blood was collected for the detection of $H$. pylori $\mathrm{IgG}$ and $\operatorname{Ig} \mathrm{A}$ antibodies. Thirty one neonates aged 1 to 30 days who were admitted in Special Care Baby Unit (SCABU) of BIRDEM Hospital were included in the study as healthy control. Only stool samples were collected from the neonates for the detection of fecal $H$. pylori antigen.

The study was approved by the Institutional Review Board and written informed consent was obtained from all cases. Consent was obtained from the guardians of the neonates for collection of fecal samples. All laboratory works were carried out in the Department of Microbiology, Ibrahim Medical College, Dhaka. The study period was from July 2012 to February 2014.

Sample preparation: After collection, blood was kept at room temperature for at least half an hour followed by centrifugation at $1500 \mathrm{rpm}$ for 10 minutes. Then the serum was separated and stored at $-20^{\circ} \mathrm{C}$. Later on the serum was used for detection of anti $H$. pylori antibodies. For stool antigen assay, the cap of the specimen collection tube was unscrewed and then the specimen collection applicator was stabbed randomly into fecal specimen in at least 3 different sites to collect approximately $50 \mathrm{mg}$ of feces. The applicator was inserted back into the tube and then the cap was tightened. Collection tube was 
shaken vigorously using vortex mixer and then centrifuged for 5 minutes at $4000 \mathrm{rpm}$. The supernatant was used for the assay.

Rapid urease test (RUT): Immediately after collection, the biopsy specimen was suspended in the rapid urease test media. Then the medium was incubated at $37^{\circ} \mathrm{C}$ and examined after 4 hours or after over-night incubation ( $24 \mathrm{hrs})$ to detect urease activity. The test was considered positive if the colour of the medium changed from yellow to pink [12, 13].

H. pylori stool antigen assay: Stool samples were analyzed for $H$. pylori antigen using ABON one step $H$. pylori antigen test device (Inverness Medical Innovation Hong Kong Limited). It is a lateral flow chromatographic immunoassay. The test was performed as per instruction of the manufacturer. Two drops of extracted stool sample was added to the sample well of the test kit. The result was read 10 minutes after dispensing the sample. A test was considered positive when a purple-pink line (test line) appeared in addition to the control line and was considered negative when only the control line appeared. Lack of control line indicated invalid result.

H. pylori IgG and IgA detection by ELISA: Serum samples were tested for the presence of anti $H$. pylori $\operatorname{IgG}$ and $\operatorname{IgA}$ antibodies. Test was performed by DRG $H$. pylori IgG and IgA ELISA kit (DRG International Inc., USA) according to manufacturer's instruction.

\section{Results}

Present study was carried out on 91 adult dyspeptic patients and 31 neonates (aged 1- to 30 days). Of 91 patients, $17(18.7 \%)$ were diagnosed as peptic ulcer and $74(81.3 \%)$ as erosion by endoscopy. HpSAg showed higher positivity (76.5\%) in ulcer cases. Overall positivity of $\mathrm{HpSAg}$ was higher (63.7\%) in comparison to RUT (42.9\%) and IgA (62.6\%) except $\operatorname{IgG}(97.8 \%)$. Out of 91 , cases, $83.5 \%$ was positive for either HpSAg or IgA (Table1). HpSAg test was compared with RUT and serology. Out of $58 \mathrm{HpSAg}$ positive cases, $67.2 \%$ were positive by RUT (Table 2 ). None of the HpSAg negative case was positive by RUT. HpSAg positive cases show higher IgA and IgG positivity than stool $\mathrm{Ag}$ negative cases. IgG was positive in all HpSAg positive cases. RUT and serology were compared with HpSAg test alone and in combination (Table 3). All the 39 RUT positive cases were also positive by HpSAg test (100\%). Out of 52 RUT negative cases, 19 (36.53\%) were stool antigen positive. All the 26 RUT and IgA positive cases were also positive for $\mathrm{HpSAg}$. We included fecal samples from 31 neonate aged 1 to 30 days as a negative control for stool antigen. It was considered that the neonates would not be exposed to $H$. pylori. Among them, 1 (3.23\%) was positive for stool antigen. The HpSAg method had a sensitivity of $100 \%$ for detection of $H$. pylori infection.

Table-1: Results of RUT, serum $\boldsymbol{H}$. pylori IgG, IgA and HpSAg tests for detection of $\boldsymbol{H}$. pylori infection in study population

\begin{tabular}{|c|c|c|c|c|c|c|c|c|}
\hline \multirow{2}{*}{ Diagnosis } & \multirow{2}{*}{$\begin{array}{c}\text { Total } \\
\text { No. of } \\
\text { case }\end{array}$} & \multicolumn{7}{|c|}{ Number (\%) positive by } \\
\hline & & RUT & HpSAg $^{\mathrm{a}}$ & IgA & IgG & $\begin{array}{c}\text { HpSAg/ } \\
\text { IgA }\end{array}$ & $\begin{array}{c}\text { HpSAg/ } \\
\text { RUT }\end{array}$ & $\begin{array}{c}\text { HpSAg/ } \\
\text { IgG }\end{array}$ \\
\hline Ulcer & 17 & $\begin{array}{l}10 \\
(58.8)\end{array}$ & $\begin{array}{l}13 \\
(76.5)\end{array}$ & $\begin{array}{l}12 \\
(70.5)\end{array}$ & $\begin{array}{l}17 \\
(100)\end{array}$ & $14(82.4)$ & $13(76.5)$ & $17(100)$ \\
\hline Erosion & 74 & $\begin{array}{l}29 \\
(39.1)\end{array}$ & $\begin{array}{l}45 \\
(60.8)\end{array}$ & $\begin{array}{l}45 \\
(60.8)\end{array}$ & $\begin{array}{l}72 \\
(97.2)\end{array}$ & $62(84.0)$ & $45(61.0)$ & $72(97.2)$ \\
\hline Total & 91 & $\begin{array}{l}39 \\
(42.9)\end{array}$ & $\begin{array}{l}58 \\
(63.7)\end{array}$ & $\begin{array}{l}57 \\
(62.6)\end{array}$ & $\begin{array}{l}89 \\
(97.8)\end{array}$ & $76(83.5)$ & $58(63.7)$ & 89 (97.8) \\
\hline
\end{tabular}

Note: HpSAg/IgA indicate either HpSAg or IgA positive; HpSAg/RUT indicate either HpSAg or RUT positive; a= $p<0.05)$, compared between ulcer and erosion cases for HpSAg test; $p<0.05$, compared between HpSAg and RUT. For HpSAg 95\% CI: 53.8\%-73.6\%. For HpSAg/IgA 95\% CI: 75.8\%-91.1\% 
Table-2: Relation of $H$. pylori stool antigen (HpSAg) detection with RUT and $H$. pylori antibodies in ulcer and erosion patients $(n=91)$

\begin{tabular}{l|c|c|c|c|c}
\hline \multirow{2}{*}{ Test } & \multirow{2}{*}{$\begin{array}{c}\text { No. } \\
\text { of }\end{array}$} & \multicolumn{4}{|c}{ Number (\%) positive by } \\
\cline { 3 - 6 } cases & RUT & IgA & IgG & $\begin{array}{c}\text { Both } \\
\text { IgA+ IgG }\end{array}$ \\
\hline HpSAg & 58 & 39 & 38 & 58 & 39 \\
Positive & & $(67.2)$ & $(65.5)$ & $(100)$ & $(67.2)$ \\
HpSAg & 33 & 0 & 19 & 31 & 17 \\
Negative & & & $(57.5)$ & $(96.8)$ & $(51.5)$ \\
\hline
\end{tabular}

Table-3: Comparison of RUT, serum H. pylori IgG and IgA with HpSAg test

\begin{tabular}{l|l|c|c}
\hline Test & $\begin{array}{c}\text { Test } \\
\text { result }\end{array}$ & $\begin{array}{c}\text { No of } \\
\text { Cases }\end{array}$ & $\begin{array}{c}\text { Positive for } \\
\text { HpSAg } \\
\text { N (\%) }\end{array}$ \\
\hline RUT & Positive & 39 & $39(100)$ \\
& Negative & 52 & $19(36.5)$ \\
IgA & Positive & 57 & $38(66.7)$ \\
& Negative & 34 & $20(58.8)$ \\
IgG & Positive & 89 & $58(65.1)$ \\
RUT + IgA & Negative & 2 & 0 \\
& Positive & 26 & $26(100)$ \\
RUT + IgG & Negative & 21 & $7(33.3)$ \\
& Positive & 39 & $39(100)$ \\
& Negative & 2 & 0 \\
\hline
\end{tabular}

\section{Discussion}

Accurate diagnosis of $H$. pylori infection is essential for the effective treatment and management of infection caused by $H$. pylori. Numerous invasive and noninvasive diagnostic tests have been developed. Each of the techniques has advantages as well as disadvantages depending on the clinical situation. In the present study, rapid immuno-chromatographic $H$. pylori stool antigen test was evaluated and compared with RUT and serology. It has been observed that the rate of positivity of RUT, HpSAg and serological tests were comparatively less in erosion compared to ulcer cases. However, when either HpSAg or IgA were considered then the rate of positivity in both ulcer and erosion cases were almost same $(82.4 \%$ and $84 \%$ ). Therefore, the sensitivity of the diagnosis increases if two tests are employed together.
All our RUT positive cases were also positive by HpSAg test. So it reveals that HpSAg test can efficiently detect $H$. pylori infection. This result matches with the findings of a similar study conducted in Kuwait University, where $52 \%$ of the patients had a positive RUT test when they used a single antral biopsy as we did [13]. Furthermore among RUT negative cases, $36.53 \%$ were $\mathrm{HpSAg}$ positive. This may be due to the fact that in the RUT, false-negative results may occur because of irregular distribution of bacteria in the gastric mucosa [14]. Several biopsy specimens are necessary for more accurate result.

It is apparent from the study that the rapid one step HpSAg assay has produced promising results for the detection of $H$. pylori antigen in stool samples. The result is comparable to another study where they found $66.7 \%$ of patients were positive for $H$. pylori stool antigens [15]. Almost all cases in our study were found $\mathrm{IgG}$ positive $(97.8 \%$ ) though many of them were negative for RUT, HpSAg and IgA. Probably, IgG was positive in those cases due to past infection or subclinical exposure to $H$. pylori. In contrast to serum $\mathrm{IgG}$, the IgA titers rise rapidly after infection and decrease if the infection is cleared $[2,3]$.

In the present study both $\operatorname{IgA}$ and $\mathrm{IgG}$ antibodies were positive in $67.2 \% \mathrm{HpSAg}$ positive cases. These cases were considered as true infection. On the other hand, $51 \%$ of HpSAg negative case were positive for both antibodies (Table 3). These cases should be very carefully diagnosed by other methods. It also appears in this study that positivity rate of IgA antibody (62.6\%) and HpSAg (63.7\%) is almost equal which is much higher than RUT (42.9\%). A comparison of HpSAg with RUT and serum IgA test was made for evaluating competence of $\mathrm{HpSAg}$ in detecting $H$. pylori infection in our study population. Serum IgG could not be considered as a diagnostic marker of active $H$. pylori infection as almost all cases were positive for IgG. On the other hand, IgA antibody could be specific for active infection with $H$. pylori [16]. In our study, both RUT and IgA positive 26 cases were also positive by HpSAg. So it reveals that HpSAg assay can efficiently detect active $H$. pylori infection. Furthermore, $33.3 \%$ cases were also positive for HpSAg among 21 both RUT \& IgA negative cases. 
Though culture is usually considered as gold standard to determine $H$. pylori infection, it is not performed in this study because of some limitations. Therefore, to determine specificity of the HpSAg test, stool samples were collected from 31 neonates. These neonates were considered as 'disease negative' because their possibility to infection by $H$. pylori was almost nil. However, out of 31 neonates stool samples, one $(3.23 \%)$ neonate was positive for $H$. pylori stool antigen test. Another study with infants found 5 out of 172 newborns $(2.9 \%)$ positive for $H$. pylori by stool antigen test at the $1 \mathrm{st}$ month of age [17]. The sensitivity of HpSAg test was thus $100 \%$ in our study. A systematic review of stool antigen test in untreated $H$. pylori infected patients reported sensitivity of $91 \%$, specificity of $93 \%$, and positive and negative predictive values of $92 \%$ and $87 \%$, respectively [18].

The rapid noninvasive immune-chromatographic HpSAg test is a quick and cost effective method to detect active $H$. pylori infection. It does not require specialized expertise and expensive laboratory facilities. In conclusion, the study has showed that $\mathrm{HpSAg}$ test can be a reliable alternative to other techniques for diagnosing active $H$. pylori infection in non treated patients with dyspepsia. It may be considered as a noninvasive first-line test for diagnosis of $H$. pylori infection in our region especially for children. The test may further be used in monitoring the therapeutic response in $H$. pylori infection.

\section{References}

1. Suerbaum S and Michetti P. Helicobacter pylori infection. $N$ Engl Med. 2002; 347: 1175-86.

2. Ahmad MM, Rahman M, Rumi AK, Islam S, Huq F, Chowdhury MF, Jinnah F, Morshed $\mathrm{MG}$, et al. Prevalence of Helicobacter pylori in asymptomatic population-a pilot serological study in Bangladesh. J. Epidemiol 1997; 7(4): 251-254.

3. Mahalanabis D, Rahman MM, Sarker SA, Bardhan PK, Hildebrand P, Beglinger C, Gyr $\mathrm{K}$. Helicobacter pylori infection in the young in Bangladesh: prevalence, socioeconomic and nutritional aspects. Int $J$ of epidemiol 1996; 25: 894-898.

4. Hazell SL, Borody TJ, Gal A, Lee A. Campylobacter pyloridis gastritis I: detection of urease as a marker of bacterial colonization and gastritis. Am J Gastroenterol 1987; 82(4): 292-296.

5. El-Zimaity HM, Segura AM, Genta RM, Graham DY. Histologic assessment of Helicobacter pylori status after therapy: comparison of Giemsa, Diff-Quik, and Genta stains. Mod Pathol 1998; 11(3): 288-91.

6. Granstrom M, Lehours P, Bengtsson C and Mégraud F. Diagnosis of Helicobacter pylori infection. Helicobacter 2008; 13(1): 7-12.

7. Howden CW, Hunt RH. Guidelines for the management of Helicobacter pylori infection. AdHoc Committee on Practice Parameters of the American College of Gastroenterology. Am J Gastroenterol 1998; 93(12): 2330-8.

8. Vilaichone RK, Mahachai V and Graham DY. Helicobacter pylori: diagnosis and management. Gastroenterol Clin North Am 2006; 35(2): 229-47.

9. Mégraud F and Lehours P. Helicobacter pylori detection and antimicrobial Susceptibility testing. Clin Microbiol Rev 2007; 20: 280-322.

10. Vaira D, Holton $\mathbf{J}$ and Menegatti M. Review article: invasive and non-invasive tests for Helicobacter pylori infection. Aliment Pharmacol Ther 2000; 14(Suppl.3): 13-22

11. Gulcan EM, Varol A, Kutlu T, Cullu F, Erkan T, Adal E, Ulucakli O, Erdama S. Helicobacter pylori stool antigen test. Indian $J$ Pediatr 2005; 72(8): 675-678.

12. Owen RJ, Collee JG, Fraser AG et al. Microbiological aspect of $H$. pylori infection. Mackie \& McCartey Practical Medical Microbiology, Cruickshank, $14^{\text {th }}$ ed. Singapore: Longmann Singapore Ltd 1996; 6: 451-456.

13. Siddique I, Al-Mekhaizeem K, Alateeqi N, Memon A and Hasan F. Diagnosis of Helicobacter pylori: improving the sensitivity of CLO test by increasing the number of gastric antral biopsies. J Clin Gastroenterol 2008; 42(4): 356-60. 
14. Ramis IB, de Moraes EP, Fernandes MS, Mendoza-Sassi R, Rodrigues O, Juliano CRV, Scaini CJ, da Silva PEA. Evaluation of diagnostic methods for the detection of Helicobacter pylori in gastric biopsy specimens of dyspeptic patients. Braz J Microbiol 2012; 43(3): 903-908.

15. Ibrahim NH, Gomaa AA, Abu-Sief MA, Hifnawy TM, El-Baseer Tohamy MA. The use of different laboratory methods in diagnosis of Helicobacter pylori infection: a comparative study. Life Sci J 2012; 9(4): 249-259.

16. Yamamoto I, Fukuda Y, Mizuta T, Fukada M,
Nishigami T, Shimoyama T. Serum antiHelicobacter pylori antibodies and gastritis. $J$ Clin Gastroenterol 1995; 21: S164-S168.

17. Baldassarre ME, Monno R, Laforgia N, Fumarola L, Fanelli M, Sgobba C, Hassan C, Panella C, Ierardi E. The source of Helicobacter pylori infection in the neonatal period. J Perinat Med 2009; 37(3): 288-92. doi: 10.1515/JPM.2009.045.

18. Gisbert JP, Pajares JM. Diagnosis of Helicobacter pylori infection by stool antigen determination: a systematic review. Am $J$ Gastroenterol 2001; 96(10): 2829-38. 